\title{
Simulation of Contaminates Around the Solid Immersion Lens in a Near-Field Optical Recording System
}

\author{
Elon Terrell and C. Fred Higgs III \\ Data Storage Systems Center, Mechanical Engineering Department, Carnegie Mellon University, Pittsburgh, PA 15213-2890 USA
}

\begin{abstract}
Accurate predictions of contamination in next-generation optical storage drives are paramount when active gap control is employed. In near-field recording devices, the read/write interface can be on the order of 20-30 $\mathrm{nm}$, which means that the gap could be quite susceptible to contamination. Predictive modeling approaches for studying the behavior of contaminates in nanoscale hydrodynamic interfaces are needed. Here, we present such a model. The interface consists of a flat disk surface translating under a solid immersion lens (SIL) of hemispherical geometry. We present the computational modeling simulation results for nano-scale contaminates around the near-field SIL. The simulation shows that the discrete contaminates actually circumnavigate the SIL/disk interface during operation. We identify and discuss the external influences on the discrete contaminate particle behavior.
\end{abstract}

Index Terms-Near-field recording, particulate contamination, solid immersion lens.

\section{INTRODUCTION}

$\mathbf{I}$ $\mathrm{N}$ this paper, the results of contaminates around a hemispherical solid immersion lens (SIL) [1]-[3] are presented. The SIL is a critical component in a near-field optical recording (NFR) system that is predicted to increase storage capacity to $300 \mathrm{~GB}$ on a removable disk [4]. One unexpected phenomenon that appears to be intrinsic with the design of this NFR system is its reported robustness against dust and contamination. While conventional air bearing slider (ABS) systems and actuated ABS systems have been known to become contaminated [5]-[7], the actuated SIL system did not show any signs of contamination. Additionally, it was reported that the system demonstrated that dust particles and contaminates actually circumnavigated the SIL/disk interface during dynamic operation of the disk. This counter-intuitive event motivated the authors to construct a multiphase (particle/fluid) computational fluid dynamics (CFD) simulations of the Philips NFR actuated-SIL inside a lens holder. The results of the discrete particle behavior in the SIL/disk interface and the leading and trailing edge regions are presented in this work.

\section{CFD Modeling Simulation}

\section{A. Overview of Modeling Domain}

All simulations were conducted using Fluent 6.2, a commercial CFD solver. The geometry of the SIL, which maintains a gap height of $h_{\text {gap }}=25 \mathrm{~nm}$ between itself and the disk, is

Digital Object Identifier 10.1109/TMAG.2006.888697

Color versions of one or more of the figures in this paper are available online at http://ieeexplore.ieee.org.

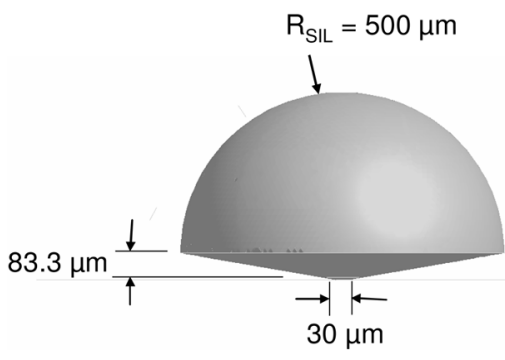

Fig. 1. Diagram of SIL geometry.

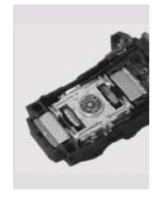

(a)

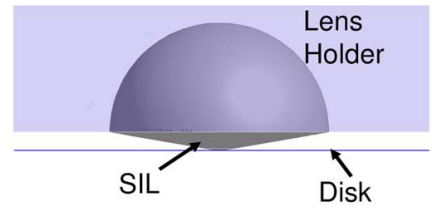

(b)
Fig. 2. (a) Image of SIL inside actuating holder (from [4]) and (b) simulated domain.

shown in Fig. 1. The upper part of the SIL is a hemisphere with a radius of $R_{\text {lens }}=500 \mu \mathrm{m}$, while the lower part of the SIL is a conical frustum with a bottom (aperture) radius $R_{\text {aperture }}=15 \mu \mathrm{m}$ and a height $h_{\text {lens }}=83.3 \mu \mathrm{m}$. As mentioned previously, this study focused on analyzing the SIL inside its actuating holder, as shown in Fig. 2.

The simulated flow domain, shown in Fig. 3, consisted of the simulated holder/lens and simulated disk separated by a volume of air. The stationary holder and lens are represented by the bordering top horizontal plane and intruding lens surface, respectively. The spinning disk was represented by the flat bottom surface of the domain. Fig. 4(a) and (b) shows the coordinate system of the domain. The $x$ and $z$ distances were measured in the streamwise and spanwise directions from the center of the 


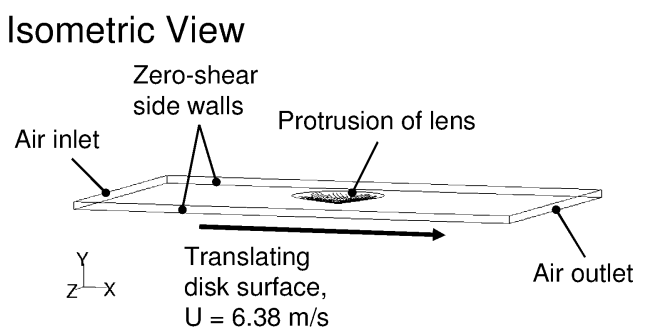

(a)

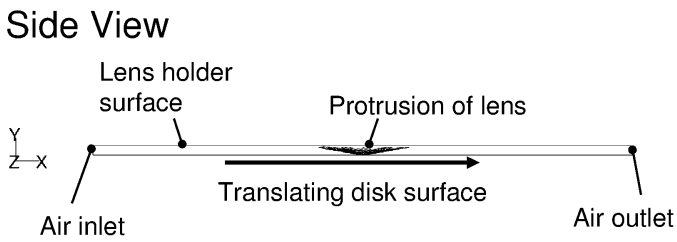

(b)

Fig. 3. Diagram of modeling domain. (a) Isometric view. (b) Side view.

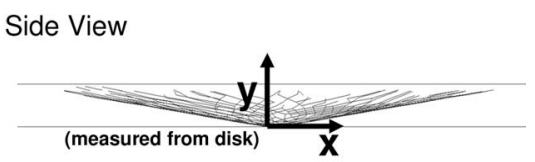

(a)

Isometric View

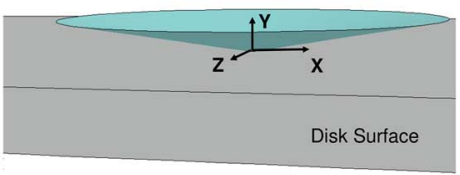

(b)

Fig. 4. Coordinate system of the modeling domain. (a) Side view. (b) Isometric view.

lens, respectively, while the $y$ distance was measured vertically across the SIL/disk gap.

While the actual SIL hovers above a rotating disk surface, the disk in this work was modeled as a translating surface with a velocity of

$$
U=(2 \pi / 60) N R_{\text {disk }}=6.38 \mathrm{~m} / \mathrm{s}
$$

where $N$ is the rotational speed of the disk, which was taken as $N=1200 \mathrm{rpm}$ [4], and $R_{\text {disk }}$ is the radial distance between the disk and lens centers $\left(R_{\text {disk }}=50.8 \mathrm{~mm}\right)$. The downstream surface of the domain was specified with a zero-gradient outflow boundary condition, while the upstream surface of the domain was specified with a fully developed Couette velocity profile, as follows:

$$
u_{\text {inlet }}(y)=U\left(1-\frac{y}{h_{\text {total }}}\right)
$$

where $h_{\text {total }}$ is the overall height of the flow domain, which is $h_{\text {total }}=h_{\text {lens }}+h_{\text {gap }}=83.325 \mu \mathrm{m}$.

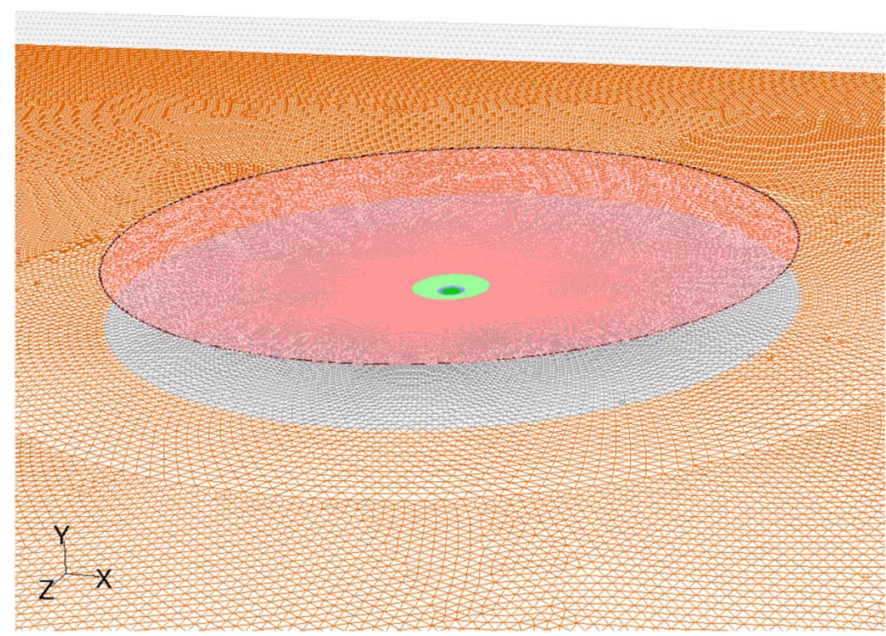

Fig. 5. Surface meshes of SIL and disk.

Additionally, the bounding surfaces at the maximum and minimum $z$-locations of the domain were modeled as slip walls with a specified normal velocity gradient of zero in order to simulate free air flow along the lateral boundaries. The remaining surfaces, including the lens, lens holder, and disk surfaces, were specified to be no-slip walls.

The three-dimensional (3-D) computational grid was created using the unstructured tetrahedral mapping scheme in Gambit, a preprocessing software package for Fluent. Samples of the surface meshes in the domain are shown in Fig. 5. The majority of the domain was meshed relatively coarsely in order to conserve computational memory. Since the gap between the disk and the lens was $h_{\text {gap }}=25 \mathrm{~nm}$, a significant amount of progressive meshing was used to discretize the SIL/disk interface and its surrounding regions. The resulting grid consisted of approximately 1855000 nodes.

\section{B. Particle-Free Flowfield Solution}

The CFD simulation of the contaminated flow was performed by first solving the particle-free flowfield and then injecting it with contaminate particles. Before the flowfield was solved, it was first characterized by calculating the maximum characteristic Reynolds number as follows:

$$
\operatorname{Re}_{h_{\text {gap }}}=\frac{\rho U h_{\text {total }}}{\mu}=34
$$

where $\rho=1.2 \mathrm{~kg} / \mathrm{m}^{3}$ and $\mu=1.86 \mathrm{e}-5 \mathrm{~kg} / \mathrm{m} \cdot \mathrm{s}$ are the density and viscosity of air, respectively. Since the calculated Reynolds number was much less than the transitional Reynolds number of $\operatorname{Re}_{\mathrm{tr}}=2000$ for internal flows [8], it was assumed that the flowfield was laminar throughout.

The particle-free flowfield was thus solved using the steadystate, laminar, 3-D solver in Fluent. The solver iteratively solved finite difference forms of the continuity and momentum equations using a second order finite difference scheme, as described in the Fluent manual [9]. The SIMPLE algorithm, which was introduced by Patankar and Spalding [10] and described in Anderson [11], was chosen as the pressure correction method for the flow solver. 


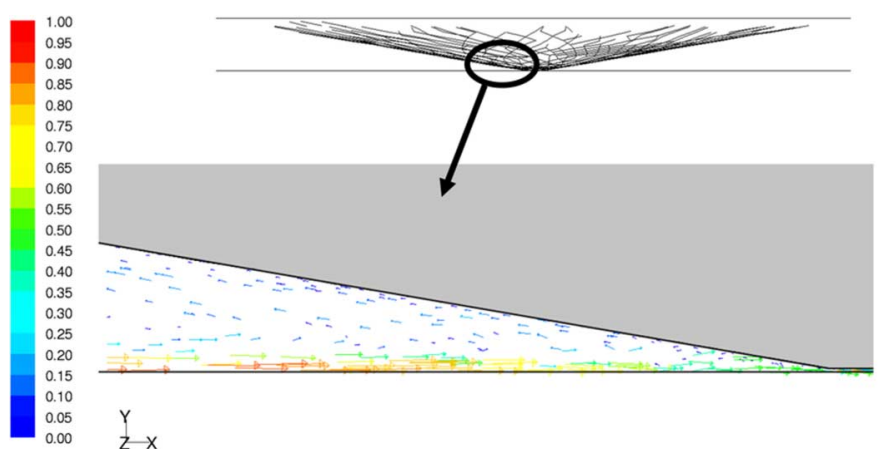

Fig. 6. Velocity vector field around SIL, colored by $u / U$.

It must be noted that the entire volume of air was assumed to behave as a continuum. The validity of this assumption is based upon the Knudsen number, which is given as follows:

$$
K n=\frac{\lambda}{L}
$$

where $L$ is the critical geometry size and $\lambda$ is the mean free path of the air molecules, which is approximately $70 \mathrm{~nm}$ for air at standard temperature and pressure. As a rule of thumb, a flowfield with $K n \ll 1$ can be treated as a continuum, while a flow with $K n \geq 1$ must be modeled by taking into account the motion of the individual air molecules. Inside the aperture gap, the length scale is $L=h_{\text {gap }}=25 \mathrm{~nm}$. Therefore, the corresponding Knudsen number would be 3.2, which implied that a continuum assumption would not be valid inside that area. However, this study mostly focused upon the flow surrounding the aperture gap, not the flow inside the gap itself. If the flowfield is observed at a radial distance larger than $r=16.5 \mu \mathrm{m}$ measured from the lens center, then the minimum feature size can be considered $L=0.25 \mu \mathrm{m}$ and larger. At such distances, the Knudsen number is $K n<0.1$ and smaller. Therefore, the continuum assumption is valid almost immediately outside the lens aperture, in the area of interest for this study.

The CFD simulations were performed using a Compaq Alphaserver node in the Pittsburgh Supercomputing Center (PSC), which contained four 1-GHz Alpha EV 6.8CB processors and 4 Gbytes of memory. The numerical solution was considered to have converged when the scaled residuals of the continuity, momentum, and energy equations approached 1e-6 or less. These convergence criteria required approximately 700 iterations, which took approximately $18 \mathrm{~h}$ on the PSC node.

The resulting velocity vectors inside the SIL/disk interface are shown in Fig. 6. As the figure shows, the flowfield follows the no-slip condition at the walls of the disk and lens, and appears to have a slight recirculation region near the lens due to the adverse pressure gradient of the air.

\section{Injection of Contaminant Particles Into Flowfield}

It was assumed that the dust and contaminates were sufficiently small and in low enough concentration for them to have a negligible effect on the air flowfield. This allowed the particle tracking to take place independent of the fluid flow calculations. Therefore, after the particle-free flowfield was solved, a series

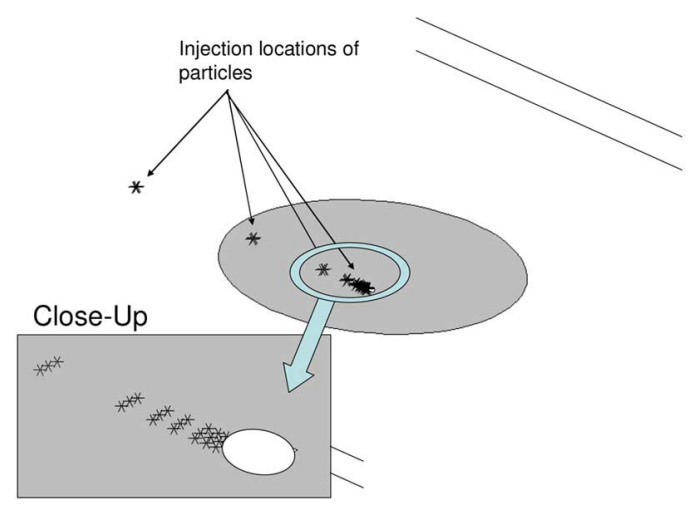

Fig. 7. Diagram showing injection locations of particles.

of simulated contaminant particles were seeded in various locations in the domain and then tracked in the Lagrangian reference frame using Fluent's discrete phase solver Each simulated nanoparticle had a diameter of $d_{p}=10 \mathrm{~nm}$ and a density of $\rho_{p}=2000 \mathrm{~kg} / \mathrm{m}^{3}$.

The seeding positions of the nanoparticles are shown in Fig. 7. All of the nanoparticles were injected at a vertical location of $y=10 \mathrm{~nm}$ above the disk surface. The nanoparticles were injected at various radial locations upstream of the lens aperture, where the injection radius $r$ (relative to the center of the SIL/disk interface) is defined as follows:

$$
r=\sqrt{x^{2}+z^{2}}
$$

The radial injection locations in this study were $r=$ $[16,20,35,60,100,200$, and $500 \mu \mathrm{m}]$. At each radial location, the particles were injected at lateral locations of $z=0,5$, and $10 \mu \mathrm{m}$.

The solver tracked each particle over a series of 4500 time steps using a Lagrangian approach. For each step in time, the solver calculated the sum of the applied forces on each particle at each time step, then updated the positions and momenta of each particle accordingly. The applied forces consisted of gravitational, buoyancy, Stokesian drag, and Saffman lift forces. The particles' interaction with solid walls was modeled by specifying a normal coefficient of restitution, $\varepsilon$, equal to zero, which means that any particle that collides with a wall surface would be inclined to "stick" to the wall unless motivated by an outside force. The Lagrangian tracking simulations required approximately 3 min per particle to complete.

\section{RESULTS}

The trajectories of the simulated nanoparticles at each radial injection location are shown in the following figures, as viewed from directly above the lens aperture. In each figure, the trajectory pathlines are colored according to $y / h_{\text {gap }}$, where $y$ is the vertical position of the particle above the disk surface. It can be observed that a particle with $y / h_{\text {gap }}$ greater than unity (1) has a vertical location that is above the lens-disk gap, and therefore may tend to be diverted around the lens or stick against the lens surface upstream of the aperture. 


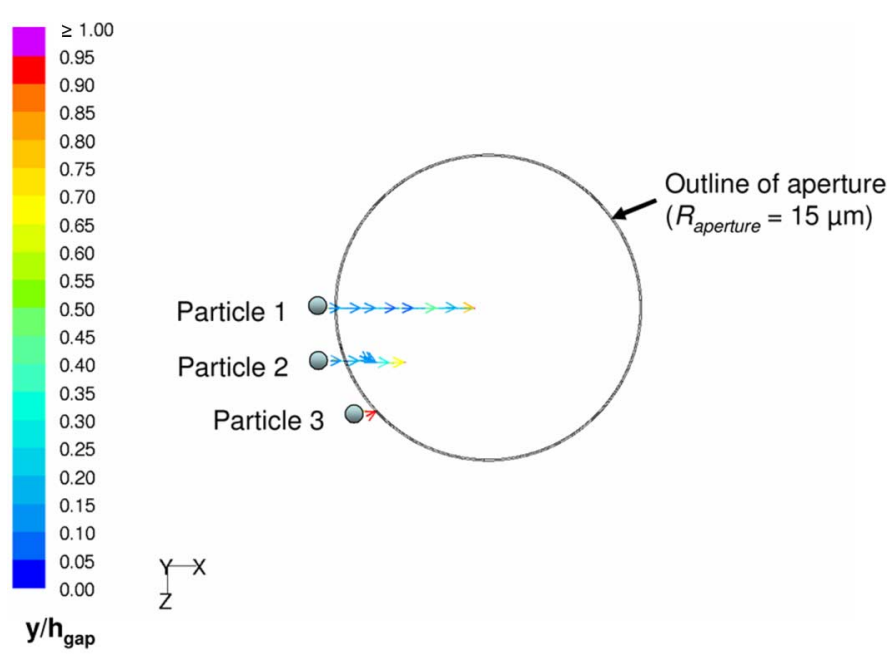

Fig. 8. Trajectory of particles that were released from $r=16 \mu \mathrm{m}$ upstream of the aperture center.

Fig. 8 shows the trajectories of nanoparticles that were released from a radius of $r=16 \mu \mathrm{m}$ upstream of the center of the lens, which is $1 \mu \mathrm{m}$ upstream of the entrance of the aperture since $R_{\text {aperture }}=15 \mu \mathrm{m}$. It must be noted that the flowfield inside this region has noncontinuum phenomena that are not captured in this model, such as boundary slip and rarefied gas flow. However, it was deemed important to analyze close-range injections in order to make sure that the particles were able to travel into the aperture interface when released from immediately upstream of the interface.

From the trajectory figure, it can be seen that Particles 1 and 2 , which were released from $z=0 \mu \mathrm{m}$ and $z=5 \mu \mathrm{m}$ at $r=16 \mu \mathrm{m}$, traveled directly into the aperture region. These close-range trajectories show that particles indeed will travel into the interface if they are not motivated by any external forces. The trajectory of Particle 3, however, shows that the particle experiences a slight amount of lift before sticking to the edge of the aperture. This sticking phenomenon is most likely due to the coefficient of restitution setting of zero.

Fig. 9 shows the trajectories of particles released from $r=$ $20 \mu \mathrm{m}$. This figure shows that all three of the particles released from $r=20 \mu \mathrm{m}$ are elevated to a higher vertical location as they approach the aperture. Particles 4 and 6 become stuck to the edge of the aperture, while Particle 5 is carried back upstream after initially approaching the aperture. This upstream motion can probably be attributed to the particle's upward movement into the recirculating region of air (see Fig. 6).

The trajectories of particles released from $r=35 \mu \mathrm{m}$ are shown in Fig. 10. From this figure, it can be observed that all three of the particles that were released from this injection radius were quickly lifted above the aperture/lens gap after being released. Particles 7 and 8 followed similar pathlines as Particles 4 and 5 (from Fig. 9), which were released from the $r=20 \mu \mathrm{m}$ radius. Particle 9 is diverted outward in a similar manner as that of Particle 6, but travels slightly around the aperture as opposed to sticking on the aperture edge.

Figs. 11 and 12 show the trajectories of Particles 10-15, which were released from $r=60 \mu \mathrm{m}$ and $r=100 \mu \mathrm{m}$

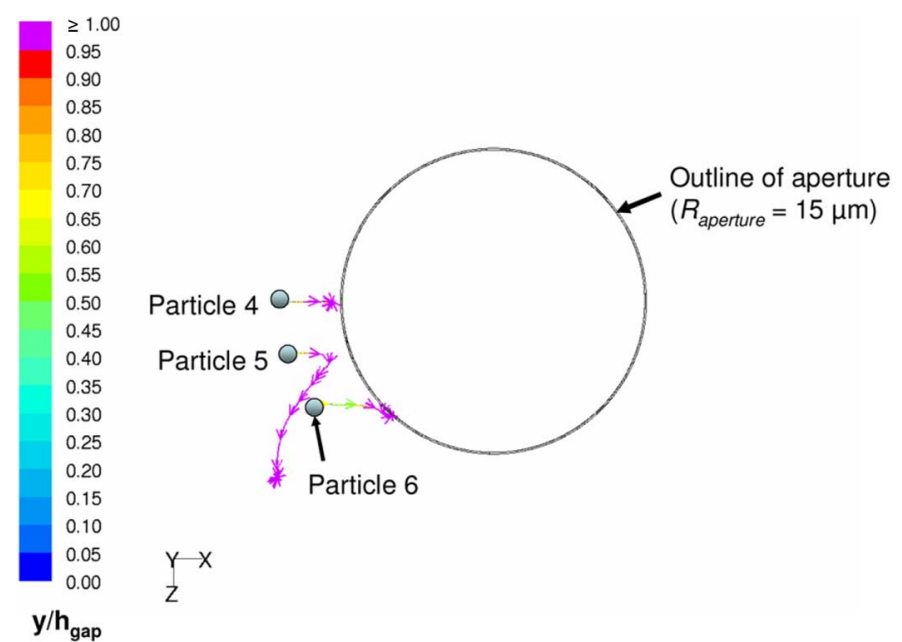

Fig. 9. Trajectory of particles that were released from $r=20 \mu \mathrm{m}$ upstream of the aperture center.

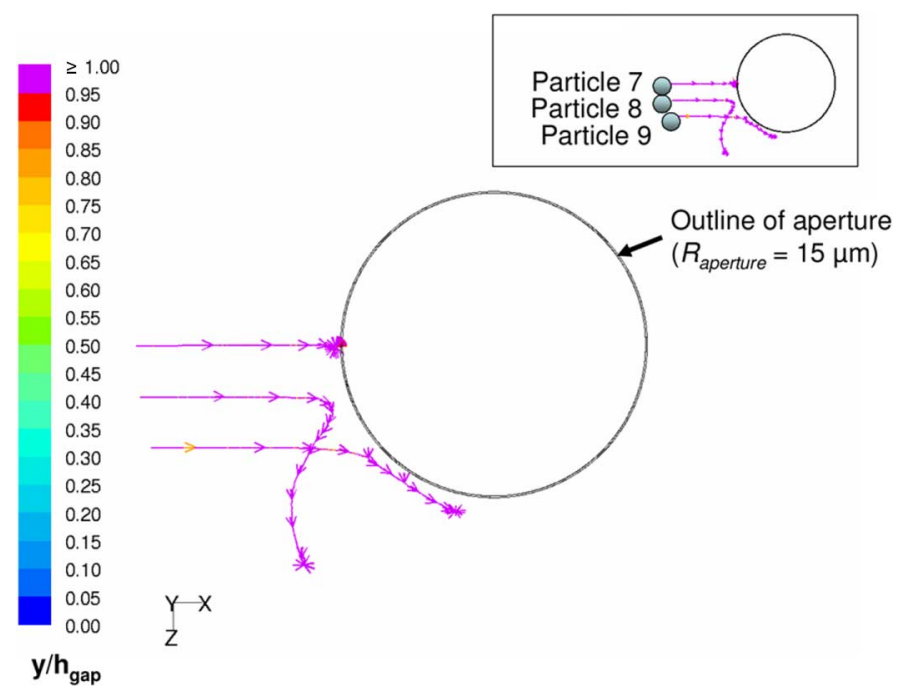

Fig. 10. Trajectory of particles that were released from $r=35 \mu \mathrm{m}$ upstream of the aperture center.

upstream of the center of the aperture, respectively. From these figures, it can be seen that Particles 10 and 13, which were both released from $z=0 \mu \mathrm{m}$, have trajectories that are diverted back upstream after initially approaching the lens aperture. This phenomenon is most likely due to the backflow in the recirculation region, as discussed previously. Particles 12 and 15 , meanwhile, both follow trajectories that slightly diverge around the aperture after being released from $z=10 \mu \mathrm{m}$, although Particle 12 is subsequently pushed back downward and then enters the aperture/disk interface. Particles 11 and 14 have a significant difference in their trajectories after being released from $z=5 \mu \mathrm{m}$, as Particle 11 is carried completely around the aperture while Particle 14 is only partially carried around the aperture.

Figs. 13 and 14 show the trajectories of particles released from $r=200 \mu \mathrm{m}$ and $r=500 \mu \mathrm{m}$, respectively. All of the particles that were released from these two radial locations were lifted up above the aperture gap height far upstream of the aperture. The particles that were released at $z=0 \mu \mathrm{m}$ became 


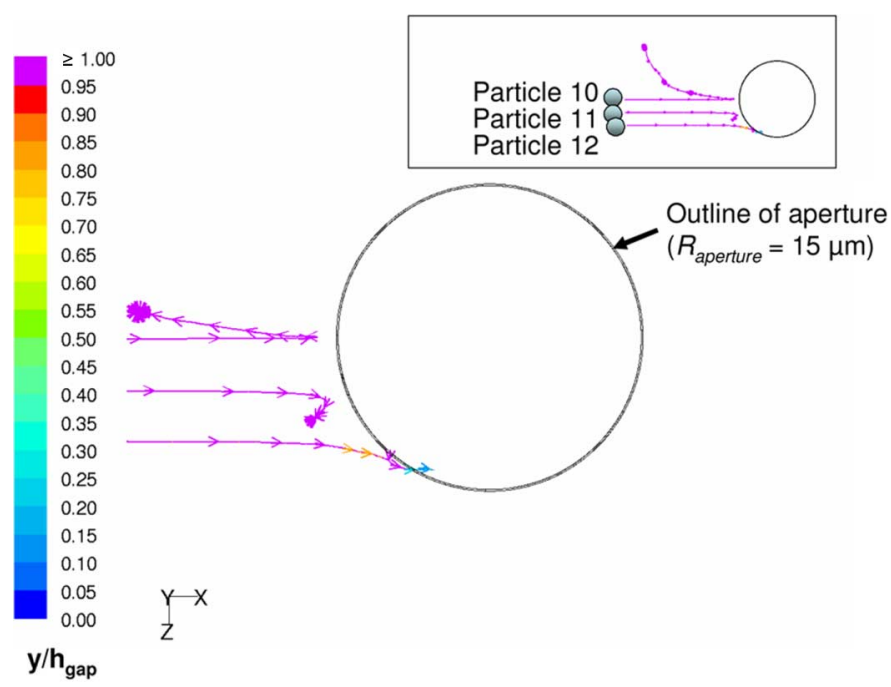

Fig. 11. Trajectory of particles that were released from $r=60 \mu \mathrm{m}$ upstream of the aperture center.

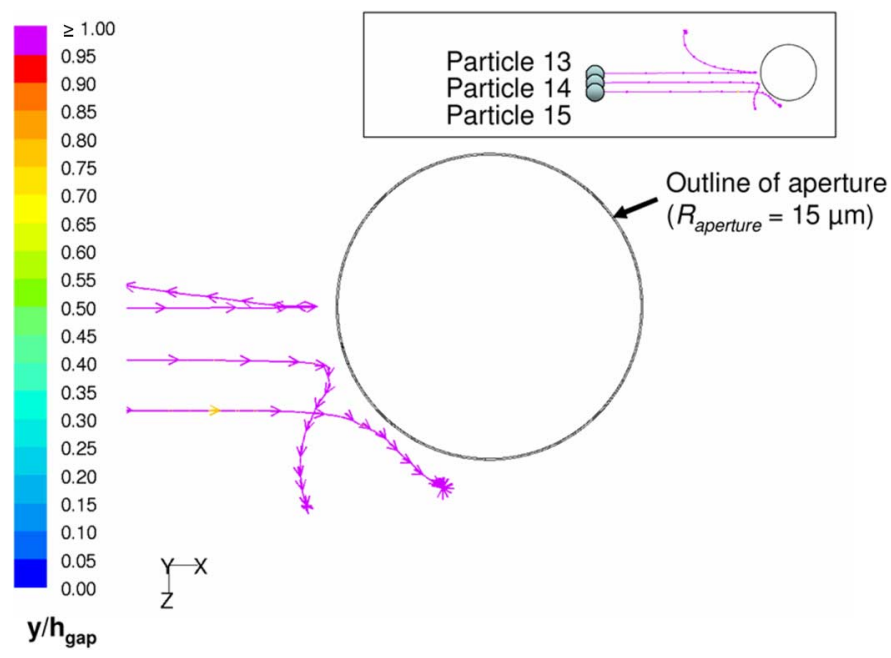

Fig. 12. Trajectory of particles that were released from $r=100 \mu \mathrm{m}$ upstream of the aperture center.

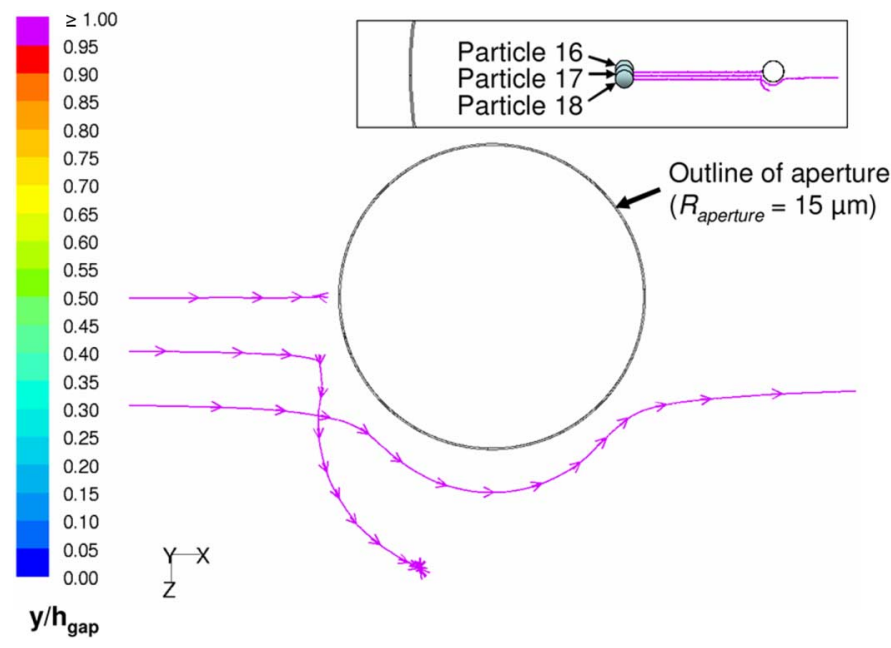

Fig. 13. Trajectory of particles that were released from $r=200 \mu \mathrm{m}$ upstream of the aperture center.

stuck on the lens surface, while the particles that were released at $z=5 \mu \mathrm{m}$ and $z=10 \mu \mathrm{m}$ were diverted around the aperture.

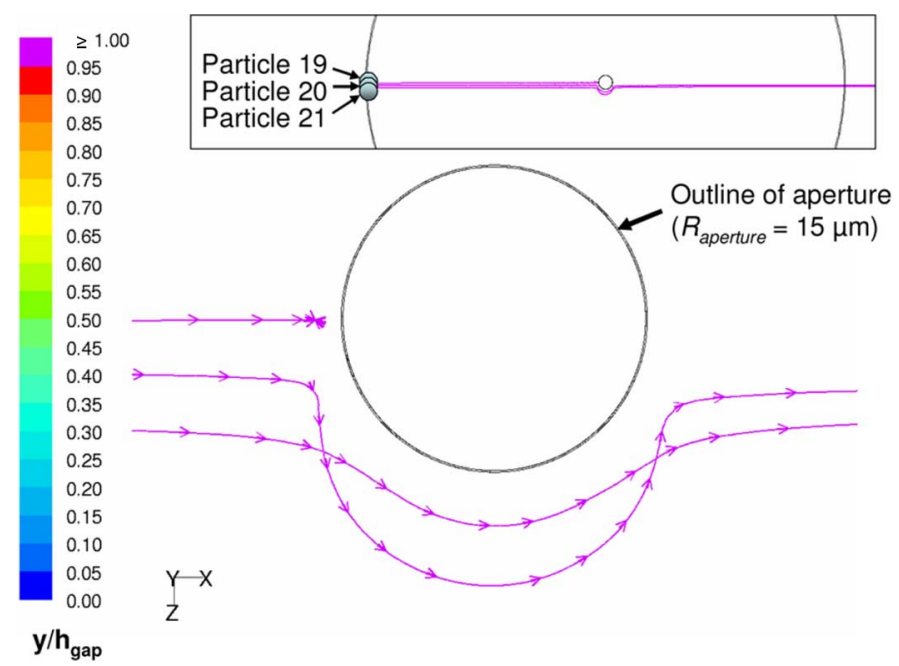

Fig. 14. Trajectory of particles that were released from $r=500 \mu \mathrm{m}$ upstream of the aperture center.

The trajectory figures show that all of the particles that avoid the aperture disk gap are first lifted up above $y / h_{\text {gap }}=1$. After being lifted above $y / h_{\text {gap }}=1$, the particles are either diverted away from the aperture by the surrounding airflow or they stick to the SIL surface upstream of the aperture. It is likely that this lifting phenomenon is a critical cause of the particles' divergence around the lens.

\section{Discussion}

The physical phenomenon behind this lifting effect can be seen by comparing the magnitude of each of the applied vertical forces on each particle as a function of the particles' downstream distance $(x)$. These forces were calculated using the output particle and fluid velocity data from Fluent. The vertical forces included, gravity, buoyancy forces, Saffman lift, and drag forces from the fluid motion. Detailed explanations of each of the applied forces are provided in past studies of contamination in magnetic data storage systems [5], [6], [12], particle flow modeling studies in tribological systems [13]-[15], as well as the Fluent 6.2 manual [9], so they are not discussed in this paper.

The primary cause of the lifting of the particles can be investigated by plotting the applied vertical $(y)$ forces on various particles as they approach the aperture. These forces are shown in Figs. 15-17 for Particles 10, 15, and 18, which were released at midplane $(z=0 \mu \mathrm{m})$ at $r=60 \mu \mathrm{m}, r=200 \mu \mathrm{m}$, and $r=500 \mu \mathrm{m}$, respectively. The magnitudes of the various forces on the particles are plotted in terms of femtonewtons (fN), which has the following unit conversion:

$$
1 \text { femtonewton }(\mathrm{fN})=10^{-15} \text { newton }(\mathrm{N}) .
$$

Recall that the injected particles each have a diameter of $d_{p}=$ $10 \mathrm{~nm}$. All of the figures show that the gravity/buoyancy forces are always relatively small, while the Saffman lift force is usually smaller than the drag force. The drag force was positive in many areas upstream of the lens due to some vertical motion of the air, which would be the reason why the particles were lifted up above the lens aperture. From a comparison of Figs. 15-17, it appears that the relative magnitude of the vertical drag force, 


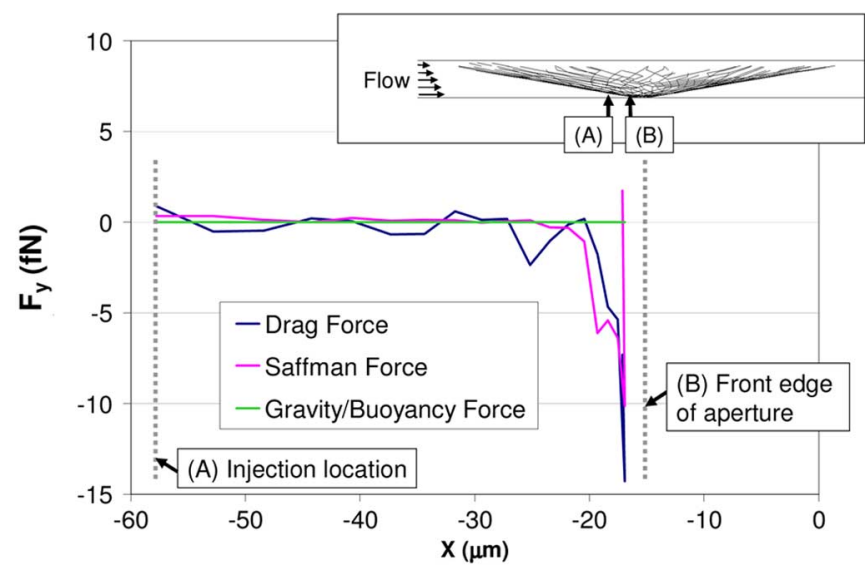

Fig. 15. Applied forces in the vertical $(y)$ direction along the horizontal $(x)$ path length for Particle 10, which was released from midplane $(z=0 \mu \mathrm{m})$ at $r=60 \mu \mathrm{m}$ upstream of the lens center.

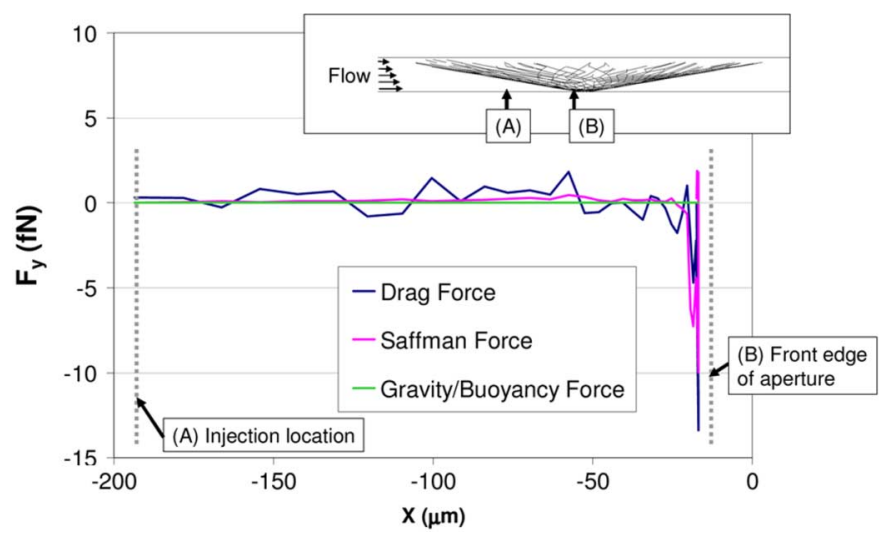

Fig. 16. Applied forces in the vertical $(y)$ direction along the horizontal $(x)$ path length for Particle 16, which was released from midplane $(z=0 \mu \mathrm{m})$ at $r=200 \mu \mathrm{m}$ upstream of the lens center.

when compared to that of the Saffman and gravity/buoyancy forces, becomes increasingly significant as the particle injection location is moved further upstream. This phenomenon is most likely caused by the decreased nominal shear rate $\dot{\gamma}=$ $U_{\text {disk }} / h_{\text {gap }}(x)$ outside the SIL/disk interface. Higher nominal shear rates attenuate the vertical force components which induce particle lift.

As mentioned previously, the results for the applied vertical forces showed that the drag force had a much greater influence on the particle trajectory than the Saffman force. This theory was confirmed by running particle tracking simulations with the Saffman force effects alternatively turned on and turned off. The particle trajectories appeared to be the same regardless of whether the Saffman force was active or inactive, which confirmed that it had little influence in this study. It must be noted that electrostatic, interparticle, and Brownian forces were neglected in this model. Their effects will be analyzed in future particle contamination studies.

Many of the trajectory figures show that the injected particles are lifted above the lens gap almost immediately after being released from its initial seeding location. The authors hypothesize that the vertical drag force which caused this initial lifting was a residual effect of the recirculation region upstream of the

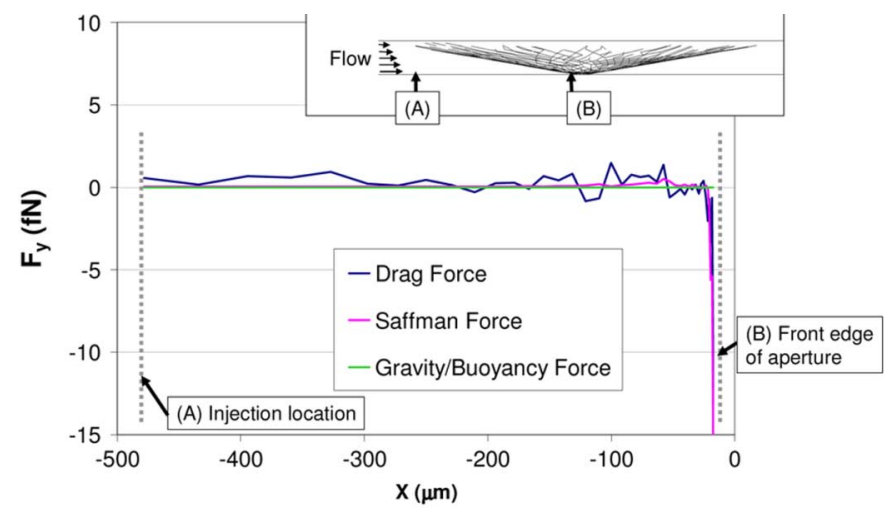

Fig. 17. Applied forces in the vertical ( $y)$ direction along the horizontal $(x)$ path length for Particle 19, which was released from midplane $(z=0 \mu \mathrm{m})$ at $r=500 \mu \mathrm{m}$ upstream of lens center.

aperture interface, as shown in Fig. 6. Since the air flow pattern around the bottom of the lens is unique to the conical SIL design, the authors rationalize that the lifting force of the particle upstream of the lens was also unique to the SIL geometry. This is one possible explanation of why the near-field solid immersion lens appears to exhibit "robustness against dust and contamination" [4], [16] while the existing ABS technologies do not.

\section{CONCLUSION}

A computational multiphase (particle/fluid) simulation was developed in order to determine the physical phenomena behind the SIL's apparent robustness against contamination. The simulation first solved for the particle-free flowfield around the lens, then seeded contaminating nanoparticles upstream of the aperture gap and tracked their trajectory using a Lagrangian approach. It was found that the contaminants were lifted above the height of the SIL interface due to the drag force caused by the air motion, and subsequently circumnavigated the SIL.

The observations from this study can be used to explain why the solid immersion lens has a reduced amount of particulate contamination when compared with conventional ABS systems. The physics behind the particle diversion phenomenon opens the possibility for redesigned ABS geometries, which can reduce particulate contamination and mitigate hard drive failure.

As mentioned previously, various applied forces on the particles were omitted from this study. These forces, which include Brownian, interparticle, and electrostatic forces, will be addressed in future work. Additionally, future studies will also examine the effect of varying particle size, as only particles with diameter $d_{p}=10 \mathrm{~nm}$ were investigated in this study.

\section{ACKNOWLEDGMENT}

The numerical computations were performed on the National Science Foundation Terascale Computing System at the Pittsburgh Supercomputing Center. The authors gratefully acknowledge the support of the Alfred P. Sloan Foundation, the Pittsburgh Supercomputer Center, and the Pennsylvania Infrastructure Technology Alliance for their support of this research. The authors would also like to thank their colleagues, E. Schlesinger and J. Bain, in the Data Storage Systems Center (DSSC) at 
Carnegie Mellon University for educational discussions on optical data storage.

\section{REFERENCES}

[1] F. Zijp, M. B. Van Der Mark, J. I. Lee, C. A. Verschuren, B. H. W. Hendriks, M. L. M. Balistreri, H. P. Urbach, M. A. H. Van Der Aa, and A. V. PadiyF. Zijp, M. B. Van Der Mark, J. I. Lee, C. A. Verschuren, B. H. W. Hendriks, M. L. M. Balistreri, H. P. Urbach, M. A. H. Van Der Aa, and A. V. Padiy, "Near field read-out of a 50 GB first-surface disk with $\mathrm{NA}=1.9$ and a proposal for a cover-layer incident, dual-layer near field system," in Proc. Optical Data Storage 2004, Monterey, CA, Apr. 18-21, 2004, pp. 209-223.

[2] T. Ishimoto, K. Saito, M. Shinoda, T. Kondo, A. Nakaoki, and M. Yamamoto, "Gap servo system for a biaxial device using an optical gap signal in a near field readout system," Jpn. J. Appl. Phys. Part 1: Regular Papers and Short Notes and Review Papers, vol. 42, pp. 2719-2724, 2003.

[3] S. J. Yoon, J. Lee, Y. P. Park, and D. H. Choi, "Design of optical flying head for magnetooptical recording," IEEE Trans. Magn., vol. 41, no. 10, pp. 2851-2853, Oct. 2005.

[4] F. Zijp, M. van der Mark, C. Verschuren, J. Lee, J. van den Eerenbeemd, P. Urbach, and M. van der AA, "High-density near-field optical recording with a solid immersion lens, conventional actuator, and a robust air gap servo," IEEE Trans. Magn., vol. 41, no. 2, pp. 1042-1046, Feb. 2005.

[5] S. C. Lin, T. C. Kuo, and C. C. Chieng, "Particle trajectories around a flying slider," Trans. ASME, J. Tribol. , vol. 120, pp. 69-74, 1998.

[6] X. Shen and D. B. Bogy, "Particle flow and contamination in slider air bearings for hard disk drives," J. Tribol., vol. 125, pp. 358-363, 2003.
[7] Q. H. Zeng, R. Pit, R. Payne, P. Baumgart, and F. Y. Huang, "Modeling and simulation of hard-particle interaction in head/disk interfaces," IEEE Trans. Magn., vol. 41, no. 2, pp. 604-609, Feb. 2005.

[8] J. A. Roberson and C. T. Crowe, Engineering Fluid Mechanics. New York: Wiley, 1997.

[9] Fluent 6.2 User's Guide. Lebanon, NJ: Fluent, Inc., 2005.

[10] S. V. Patankar and D. B. Spalding, "A calculation procedure for heat, mass, and momentum transfer in three dimensional parabolic flows," Int. J. Heat Mass Transfer, vol. 15, p. 1787, 1972.

[11] J. D. Anderson, Computational Fluid Dynamics: The Basics with Applications. New York: McGraw-Hill, 1995.

[12] S. Zhang and D. B. Bogy, "Effects of lift on the motion of particles in the recessed regions of a slider," Phys. Fluids, vol. 9, p. 1265, 1997.

[13] E. J. Terrell, V. K. Jasti, and C. F. Higgs III, "Modeling the motion of slurry nanoparticles during chemical mechanical polishing," in Proc. Int. Mechanical Engineering Congr. Expo., Orlando, FL, 2005.

[14] E. J. Terrell, J. I. Garcia, and C. F. Higgs III, "Two-phase hydrodynamic modeling of particulate fluids in sliding contacts," in Proc. World Tribology Conf., 2005.

[15] E. J. Terrell and C. F. Higgs III, "A modeling approach for predicting the abrasive particle motion during chemical mechanical polishing," $J$. Tribol., 2007, accepted for publication.

[16] T. Ishimoto, T. Ito, M. Shinoda, K. Saito, T. Kondo, T. Matsui, S. Kim, A. Nakaoki, and M. Yamamoto, "Head analysis in air flow on nearfield optical disk system with 2-axis actuator," Jpn. J. Appl. Phys., Part 1: Regular Papers and Short Notes and Review Papers, vol. 44, pp. 3410-3411, 2005.

Manuscript received May 6, 2006; revised November 21, 2006. Corresponding author: F. Higgs (e-mail: higgs@andrew.cmu.edu). 